\title{
Variation in Neutrophil Levels and Artemisinin-Based Combination Therapy Efficacy in West-africa
}

\author{
Moussa Djimde \\ MRTC: Malaria Research and Training Center \\ Japhet Kabalu Tshiongo \\ University of Kinshasa: Universite de Kinshasa \\ Kassoum Kayentao \\ MRTC: Malaria Research and Training Center \\ Bakary Fofana \\ MRTC: Malaria Research and Training Center \\ Charles Arama \\ MRTC: Malaria Research and Training Center \\ Siriman Sodiomo \\ Groupe de Recherche Action en Santé \\ Jean Bosco Ouedraogo \\ Institut des Sciences et des Techniques Bobo Dioulasso \\ Abdoul Habib Beavogui \\ Centre National de Formation et de Recherche en Santé Rurale \\ Issaka Sagara \\ Malaria Research and Training Center \\ Alassane Dicko \\ Malaria Research and Training Center \\ Petra F. Mens \\ Amsterdam University Medical Centres: Amsterdam Universitair Medische Centra \\ Henk D. F. H. Schallig \\ Amsterdam University Medical Centres: Amsterdam Universitair Medische Centra \\ Abdoulaye Djimde ( $\nabla$ adjimde@icermali.org ) \\ MRTC-USTTB https://orcid.org/0000-0003-0062-2283
}

\section{Research}

Keywords: Malaria, Neutrophils, artemisinin-based combination therapy, West Africa

Posted Date: November 2nd, 2020

DOI: https://doi.org/10.21203/rs.3.rs-100164/v1

License: (c) (i) This work is licensed under a Creative Commons Attribution 4.0 International License. Read Full License 


\section{Abstract}

Background

White blood cells, including abundant neutrophils, constitute the first line of innate defence against pathogens. Neutrophils are involved in pathogen clearance by phagocytosis. However, their role on malaria parasite clearance and on the efficacy of Artemisinin-based Combination Therapy (ACT) are poorly understood.

Methods

In a prospective longitudinal in vivo study, conducted from 2010 to 2014 in Mali, Burkina Faso and Guinea, in which 5360 malaria cases were enrolled, neutrophil rates were compared with malaria carriage after treatment with different ACTs. i.e. Artemether - lumefantrine (AL), Artesunate - amodiaquine (ASAQ), Dihydroartemisinin - piperaquine (DHAPQ) or Pyronaridine artesunate (PA). Depending on the level of neutrophils in the blood, study cases were classified as having neutropenia, normal neutrophils level or neutrophilia. A secondary analysis on data of 4845 cases was performed using R ggplot2 statistical package to compare the post treatment neutrophil levels means and standard deviation between different treatment arms at subsequent malaria episodes.

Results

Microscopy showed that at day 7 only 4 patients in the neutropenia group and treated with AL had a malaria positive blood smear. At Day 28, rate of recurrent parasitemia in the $\mathrm{AL}$ arm was significantly higher in cases with neutropenia (50.9\%) versus in cases with normal rates of neutrophils $(43.1 \%)$ and in patients with neutrophilia $(6 \%)(p<0.001)$. For patients treated with ASAQ, the rate of recurrent parasitemia was $58.8 \%$ in the neutropenia group versus $29.4 \%$ in patients with normal rates of neutrophils and $11.8 \%$ in patients with neutrophilia ( $p<0.001$ ). No patient treated with DHAPQ with normal neutrophil counts or neutrophilia was carrying malaria parasites at Day 28 . Compared to adults, under 5 years old children were shown to be at risk of malaria parasite reappearance at post treatment Day $28(\mathrm{OR}=5.64, \mathrm{p}=0.04)$. Regardless of the ACT used in this study, we observed a significant decrease of neutrophil levels at day 3 post-treatment. Furthermore, regardless the ACT used, an increase in the prevalence of gametocytemia was found in the neutropenic group.

Conclusion

Patients with neutropenia had higher rates of recurrent parasitemia after artemisinin-based combination therapy. Parasite recurrence in patients with neutropenia was faster after treatment with AL.

\section{Background}

In healthy individuals, polymorphonuclear neutrophils (PMN), which are the most important component of the white blood cells (WBC), play an important role in the host's immunological response against bacterial, fungal and malaria infections because of their capacity to phagocytose pathogens [1,2]. In addition, they contribute to the recruitment of both nonspecific and specific immune effector cells [3]. Finally, since WBC and PMN respond to produced cytokines [4,5], they also modulate the balance between humoral and cell-mediated immunity by contributing to the promotion of T helper cells (i.e. TH1 or TH2) response [6]. PMNs are engaged in a complex cross-talk with immune and endothelial cells that bridges innate and adaptive immunity.

In some individuals' neutrophil levels may be lower than normal i.e. neutropenia, which and can be acute, congenital or cyclic. Neutropenia, defined as an absolute decrease in the number of neutrophils circulating in the blood [7], increases the risk of infection, in particular from pyogenic and enteric bacteria. In addition, the risk of fungal infections increases further when neutropenia is severe and prolonged [8]. Acute neutropenia, the resolvable short-term drop in absolute neutrophil counts due to an infection or lesion, is much more common than congenital or cyclic neutropenia [9]. Neutropenia is known to be quite common in African populations [10,11] and a genetic deletion of the Duffy antigen receptor for chemokines (DARC-null genotype) is likely to be a major determinant for neutropenia [11]. In West Africa, more than $90 \%$ of the population is believed to be Duffy antigen negative [12].

In analogy to the way PMN can control mycobacterial infection by phagocytosis of pathogens at the site of infection [13], these cells could act with similar activities against malaria infection. PMN are known to phagocytose infected Red Blood Cell (iRBC) in vivo [14]. It has been shown that neutrophils can phagocytose merozoites and occasionally trophozoites in bone marrow [15]. Neutrophils can also clear pathogens by respiratory burst called Reactive Oxygen Species (ROS). Malaria parasites growth inhibition by ROS occurs during the intra-erythrocytic development stage [16]. Furthermore, it has been demonstrated in vitro that neutrophils from children infected with Plasmodium are better able to inhibit parasite growth than neutrophils from uninfected subjects [17]. Besides the asexual forms of the malaria parasite, neutrophils can phagocyte extracellular gametocytes, while, intra-erythrocytic gametocytes are not very susceptible to neutrophils [18]. 
Artemisinin-based combination therapy (ACT) is now for almost two decades recommended by the World Health Organization (WHO) as the first-line treatment of uncomplicated falciparum malaria [19]. However, the efficacy of ACTs is an ongoing concern , due to the emergence of parasite resistance to artemisinins, [20]. Furthermore, the relationship between malaria, ACT treatment and neutrophils is largely understudied. In a previous analysis, using data from 7 randomized trials conducted in 9 countries assessing the efficacy of artesunate-amodiaquine, amodiaquine mono-therapy, artesunate mono-therapy, artemether-lumefantrine, artesunate and sulphadoxine-pyrimethamine, and dihydroartemisinin, it was found that neutropenia was frequently recorded as an adverse event at a rate of $11 \%$ [21].

Despite the role neutrophils play in innate immunity, their role in the immune response against malaria infections has been little studied. In particular, there are no extensive studies on the role neutrophils play in parasite clearance [22].

The aim of the present study was to assess the impact of neutrophil levels on the in vivo efficacy of four frequently used artemisinin-based combinations: Artemether lumefantrine (AL), Artesunate amodiaquine (ASAQ), Dihydroartemisinin piperaquine (DHAPQ) and Pyronaridine artesunate (PA).

\section{Methods}

\section{Study sites and study participants}

This is a secondary analysis of the WANECAM study [23], which was a clinical trial involving 5360 volunteers from West-Africa assessing efficacy and safety of several ACTs. The original WANECAM study was a randomised, multicentre, open-label, longitudinal clinical study carried out across seven study sites in three countries: Burkina Faso, Guinea, and Mali (http://www.wanecam.org/fr/clinical-study-protocol/) [23]. Briefly, study cases were enrolled and followed up over 2 years for consecutive malaria episodes between October 24, 2011, and February 1, 2016. Patients were treated either with AL, ASAQ, DHAPQ or PA at recommended doses. Patients, irrespective of their gender (age $\geq 6$ months and body weight $\geq 5 \mathrm{~kg}$ ), were eligible for enrolment in the study, if they had acute uncomplicated microscopically confirmed Plasmodium sp. malaria with a parasite density <200 000 parasites per $\mu \mathrm{L}$ blood, and with fever or a history of fever within 24 hours and Haemoglobin (Hb) concentration $>5 \mathrm{~g} / \mathrm{dL}$ [23]. Before enrolment, all adult study cases provided informed consent after being informed on the aims, benefits and constraints of the study in their own language. For children (< 18 years), the informed consent was obtained from parents or legal guardians after the consent taker was satisfied that the child is willing to participate. Ethical review and clearance for this project was provided by the respective ethical review boards.

\section{Neutrophils status}

Blood samples were collected from patients from an alcohol-cleaned peripheral vein into sterile venous blood collection tubes with ethylene diamine tetraacetic acid (EDTA). Blood counts, including neutrophils, lymphocytes, WBC and Hb were measured immediately at enrolment using ABX Pentra 60 - HORIBA (Montpellier, France) [24]. Blood count measurements were also performed at day 3, 7 and 28 after treatment. Neutrophils status was defined on the basis of the following cut-offs [25]: 1) neutropenia: patients who had a neutrophil count below 40\%; 2 ) normal: neutrophils level between $40 \%$ and $60 \%$; 3 ) neutrophilia: neutrophil count above $60 \%$.

\section{Drug efficacy}

During the first two days after inclusion, finger-prick blood was taken every 12 hours to make thick and thin smears to determine the presence and number of malaria parasites. Subsequently, study cases were seen on days $3,7,14,21,28,35,42$ and on days of recurrent illness for clinical examination and finger-prick for thick and thin smears, which were stained with $10 \%$ Giemsa for 15 minutes. Parasites density was determined by double reading of the thick blood smear using an Olympus CX21 microscope (Tokyo, Japan). The discordance threshold was set at $50 \%$. Discordant results were subjected to a third independent reading. Asexual parasites were counted against 200 leukocytes (in accordance with WHO standards [26]). If the number of parasites was less than 10 parasites per 200 leukocytes, the parasite count was extended to 500 leukocytes. Parasitaemia was estimated by reporting the number of parasites per microliter of blood based on the count of 8,000 leukocytes. Non-PCR corrected drug efficacy results were used in this study. Pre-treatment and days 3,7 and 28 neutrophils status and parasitaemia carriage were used to evaluate drug efficacy.

\section{Statistical analysis}

Frequency of patients with neutropenia, normal neutrophils levels and neutrophilia was computed and descriptive statistics were calculated (percentages, median and quartiles). Chi-square and Fisher tests were used to compare proportions. The threshold of 0.05 was used as statistical significance. Bar plots were used to assess the changes in gametocyte prevalence during follow-up after the administration of antimalarial drugs. Logistic regression analyses were performed to predict the effect of neutrophils status, treatment arms, lymphocytes levels, $\mathrm{Hb}$ levels, patients gender and age categories on the efficacy of the treatment. Using 'meta' R package (version 4.13-0), a forest plot was 
performed to investigate the association among socio-demographic parameters and treatment used on gametocytes reappearance at Day 3 according to neutrophils variation.

The R@ software version 3.5.1 (with aod, agricolae, ggplot2, dplyr, plotly, Hmisc, corrr, GGally, meta, and Rmisc libraries) was used for the statistical analysis.

\section{Results}

\section{General study characteristics}

Data of 4845 patients were used for this secondary analysis. The data of 515 participants were not used because neutrophil counts of these cases were not recorded. The study cases were distributed amongst the treatment arms as follows: 1163 were treated with PA, 1154 with DHAPQ, 961 with ASAQ and 1567 with AL (Table 1). With respect to gender balance, 2489 (51.4\%) of patients were male and 2356 (48.6\%) were female $(p=0.7476)$. A total of 2334 patients were enrolled in Mali, 1739 in Burkina and 770 in Guinea. Children ( $n=2981)$ with age between 5 and 14 years were the most represented (61.5\%) in the study (Table 1$)$.

The neutrophil group with the highest frequency was the neutrophilia category in Mali with 905 cases (38.8\%) and Burkina Faso with 735 cases (42.3\%), respectively. In contrast, there were more patients with neutropenia $340(44.0 \%)$ in Guinea. The median and interquartile range (IQR) of patient's bodyweight was significantly higher in neutrophilia category $24.5 \mathrm{~kg}$ (IQR, 17.6-37.8), compared to patients in neutropenia (19.6 kg, $\mathrm{IQR}=14.0-28.8)$ and normal neutrophils levels $(21.8 \mathrm{~kg}, \mathrm{IQR}=15.8-32.7)(\mathrm{p}=0.0011)$. Furthermore, the patient's median temperature was higher in neutrophilia group with $38.2^{\circ} \mathrm{C}$ (IQR, 37.4-38.8) (table 1). The prevalence of gametocytemia was significantly higher in patients with normal neutrophils level with 133 cases $(40.6 \%)$ compared to those with neutropenia and neutrophilia $(p<0.0001)$.

There was also a significant difference in patients with neutropenia in the amount of trophozoites at baseline level. These patients had a significantly lower parasite count compared with the other groups $(p<0.0001)$ (table 1$)$.

The median WBC count was significantly higher in patients with neutrophilia 8.0 (IQR, 6.3-10.6) compared to subject in neutropenia 6.5 (IQR, 5.08.6) and normal neutrophils levels 6.9 (IQR, 5.2-8.7) ( $p<0.0001)$. In contrast, the median lymphocytes count and interquartile range was significantly higher in patients with neutropenia 48.6\% (IQR, 42.92-53.7) compared to those with normal or high neutrophil counts with respectively $32.6 \%$ (IQR, 27.0-38.2) and 15.5\% (IQR, 8.1-20.3) $(\mathrm{p}<0.0001)$ (Table 1).

\section{ACTs efficacy according to neutrophils count variations}

The dynamics of malaria parasite carriage after treatment according to neutrophil status is presented in Table 2. Irrespective of the type of malaria drugs used in this study, patients with neutropenia had a significantly higher malaria parasite carriage frequency up to day 28 after treatment compared to cases with normal neutrophils level and subjects with neutrophilia $(p<0.0001)$. However, patients with neutropenia had at baseline the lowest parasite density compared to the other groups (Table 1). Microscopy revealed that at day 7 only patients in the neutropenia group, and treated with $\mathrm{AL}$, had a positive blood smear (Table 2). Data from day 28 showed that the chance of parasite recurrence is higher in patients with neutropenia regardless of the type of ACT used for treatment in this study (Table 2).

Fig. 1 shows that, irrespective of the treatment arm, the pre-treatment prevalence of gametocytemia was higher in patients with normal neutrophils level compared to neutropenia and neutrophilia groups ( $p<0.0001)$. Three days after antimalarial drug administration, and regardless of the type of ACT used, the data show an increase in the prevalence of gametocytemia in the neutropenic group while gametocyte clearance is observed in patients with normal neutrophil counts and those with neutrophilia (Fig. 1). On day 28, after treatment, only patients with neutropenia and treated with ASAQ, DHAPQ and PA carried gametocytes (Fig. 1). A forest plot (Fig. 2) shows that the ACT used (AL: OR = 0.80 [0.26; 2.46], ASAQ: OR $=1.73$ [0.85; 3.52], DHAPQ: OR $=0.99$ [0.47; 2.06], PA: OR $=0.97$ [0.45; 2.06$]$ ) was not associated with a risk of post treatment Day 3 gametocytemia. This figure also shows that neither the country of residence (Burkina Faso: OR = 0.88 [0.46; 1.68$]$, Guinea: OR = 0.29 [0.07; 1.19], Mali: OR = $1.51[0.87 ; 2.61]$ ), nor age category (under 5 years old children: $\mathrm{OR}=0.48$ [0.09; 2.53$]$, teenager: OR = $0.56[0.24$; 1.26]) was associated with a risk of gametocytemia 3 days after treatment in patients with neutropenia compared to those without neutropenia.

\section{Secondary neutropenia after ACTs administration}

At the first malaria episode (Fig. 3 A), neutrophil levels on follow-up days 3,7 and 28 were significantly lower ( $p<0.001$ ) compared to pretreatment neutrophil levels irrespective of the antimalarial treatment received by the patients. This decrease in neutrophils levels was more pronounced at day 3 after treatment. Regardless of the ACT used, the overall neutrophil level was $52.70 \%$ before treatment and decreased to 33.44 on day 3 after treatment $(p<0.0001)$. Seven days after treatment, the level of neutrophils increased significantly $(p<0.001$ regardless of treatment arm), but without reaching the threshold of normality and subsequently decreased again significantly ( $p<0.001$ regardless of treatment arm) from day 7 to day 28 (Fig. 3). 
During consecutive malaria episodes (episode 2: Fig. 3 B, episode 3: Fig. 3 C and episode 4: Fig. 3 D), the dynamics of neutrophil levels followed the same trend as in the initial episode (Fig. 3 A). Irrespective of the malaria episodes, three days after drug administration the neutrophil counts were higher in the ASAQ treatment arm $(p<0.0001)$ (Fig. 3).

\section{Association between neutrophil count variations and reinfection parasitemia after treatment}

Logistic regression analysis (Table 3 ) showed that neither being in the neutrophilia category $(\mathrm{OR}=1.57, \mathrm{p}=0.6771)$ nor having normal neutrophil levels $(\mathrm{OR}=0.97, \mathrm{p}=0.9471)$ was associated with a risk of malaria parasite reappearance at Day 28 after treatment compared to patients with neutropenia.

Other factors were found to be associated with malaria parasite reappearance during post treatment follow-up (Table 3). Compared to AL treatment arm, ASAQ (OR = 0.27, $p=0.0024)$, DHAPQ $(O R=0.07, p<0.0001)$ and PA $(O R=0.13, p<0.0001)$ showed a protective effect against malaria parasite reappearance. Each increase of lymphocyte count $(O R=0.93, p=0.0063)$ was observed to be associated with a decrease of the probability of post treatment Day 28 parasitaemia. Compared to adults, children under 5 years $(O R=5.64, p=0.0393)$ were at risk of malaria parasite reappearance at Day 28 of follow-up.

\section{Discussion}

This study showed patients with neutropenia have lower baseline parasitaemia, a higher rate of recurrent parasitaemia and higher rate of gametocyte carriage by day 28 post- artemisinin-based combination therapy compared to patients with normal or high neutrophil levels. These differences were found to be independent of the type ACT used in this study. The baseline malaria parasite density was lower in patients with neutropenia. This finding is in line with the findings of Olliaro et a/ who showed that during acute uncomplicated malaria the increase of neutrophils is positively associated with parasitaemia [27]. It has also been reported that in semi-immune travellers neutrophil counts increase with the severity of malaria infection compared to those with uncomplicated malaria [28]. An important finding form this study with regards to transmission potential of malaria is the fact that the prevalence of post treatment gametocytemia was higher in patients with neutropenia regardless of treatment arm. Neutrophils constituting the most important component of the leukocyte population [2] plays an important role in malaria infection due to its phagocytic activity [1]. The role of neutrophils in the phagocytosis of extracellular gametes has been previous described [18], and this could explain the almost total absence of gametocytes in post treatment follow-up in subjects with neutrophilia. This observation could also be due to faster clearance of asexual parasites in patients with neutropenia, which would leave very little time for differentiation into sexual stages [29].

Approximately one third of the patients had neutropenia and these results support previous studies indicating that neutropenia is quite common in African populations [10,11] where more than $90 \%$ of the African population are Duffy antigen negative [12] and that the genetic deletion of the Duffy antigen receptor is a major determinant for neutropenia [11].

In addition, the study provides the evidence of secondary neutropenia when uncomplicated malaria is treated with these four ACT regimens. In this study a secondary neutropenia is observed three days after the administration of antimalarial drugs, regardless of the treatment arm. It has been previously described that $70-90 \%$ of acute and severe neutropenia has been shown to be attributable to drugs [30]. Several medications including antimalarial drugs are described to be associated with the decrease of neutrophils levels [30,31]. A larger analysis including data from 7 clinical trials carried out in 9 countries comparing the changes in haematologic parameters of ASAQ to AQ mono-therapy, AS mono-therapy, $A L, A S+S P$ and $D H A$, revealed $11 \%$ of post treatment neutropenia [21]. A study conducted in Ghana to compare the efficacy of $A S A Q$ versus $A L$ in children showed that $3,7,14$ and 28 days post treatment neutrophil counts were significantly lower $(p<0.01)$ compared to day 0 counts in both treatment arms [32]

The present study revealed that of all four drug combinations used in this analysis, AL is the least protective against parasites carriage during 28 days' post treatment follow-up in patients with normal neutrophil levels. Artemether with a half-life of two to three hours is rapidly eliminated from plasma whereas lumefantrine is eliminated more slowly [33]. The combination allows a rapid clearance of parasitaemia but does not prevent new infections. In DHAPQ combination, piperaquine is characterized by a slow absorption and long half-life, which might prevent new Plasmodium infections [34]. While the half-life of amodiaquine is about 5 hours [35,36], the combination AS+AQ presented a protective effect in the multivariate model. Pyronaridine is described to have a mean half-life of $194.8 \pm 47.8$ hours [37].

Furthermore, the present research showed that compared to AL treatment arm, patients treated with ASAQ, DHAPQ or PA were less likely to carry gametocytes post treatment. A meta-analysis of individual patient data demonstrated that the appearance of gametocytes in patients' blood was lowest after AL treatment and significantly higher after DHAPQ (adjusted hazard ratio (AHR), 2.03; $95 \% \mathrm{Cl}, 1.24-3.32 ; \mathrm{p}=0.005 \mathrm{compared}$ to $\mathrm{AL}$ ) and ASAQ (AHR, 4.01; $95 \% \mathrm{Cl}, 2.40-6.72 ; \mathrm{p}<0.001$ compared to $\mathrm{AL}$ ) [38]. A previous study conducted in Bougoula-Hameau, Mali, to investigate the differential infectivity of gametocytes after artemisinin-based combination treatment, revealed that the Day 3 gametocytes prevalence was lower in AL arm compared to ASAQ and ASSP arms [29]. This could be explained by the fact that unlike AL which is rapidly 
eliminated from the patient's blood, the other combinations used in this study would remain at sub-therapeutic levels for a longer time promoting the production of gametocytes.

A limitation of the present study is the absence of parasitaemia data six or eight hours after treatment. Such data would have permitted the precise measurement of parasite clearance time and the slope half-life of parasite clearance using the WorldWide Antimalarial Resistance Network (WWARN) Parasite Clearance Estimator (PCE) [39] and relate these to patients' neutrophils count variations. In addition, the lack of a Duffy antigen is a limitation to determine the share of benign ethnic neutropenia [11].

A major strength of this study is the participation of several countries to represent West Africa, which makes this study relevant for a large population in Africa. In addition, the large sample size allowed this study to have sufficient power to detect even small differences or effects. This study effectively compared the four major anti-malaria drugs in relation to neutropenia and parasite recurrence.

This study suggests that neutropenia could ultimately decrease the efficacy of ACTs in West Africa. The immunological mechanisms involved warrant further investigations.

\section{Abbreviations}




\begin{tabular}{|c|c|}
\hline ACT & Artemisinin-based Combination Therapy \\
\hline$A L$ & Artemether lumefantrine \\
\hline AMC & Academic Medical Centre at the University of Amsterdam \\
\hline$A S+A Q$ & Artesunate amodiaquine \\
\hline CNRFP & Centre National de Recherche et de Formation en Santé Rurale \\
\hline $\mathrm{DHA}+\mathrm{PQ}$ & Dihydroartemisinin piperaquine \\
\hline EDTA & Ethylenediaminetetraacetic acid \\
\hline $\mathrm{g} / \mathrm{dL}$ & Gram per decilitre \\
\hline GRAS & Groupe de Recherche Action en Santé \\
\hline $\mathrm{Hb}$ & Haemoglobin \\
\hline INSTech & Institut des Sciences et des Techniques \\
\hline IQR & Interquartile range \\
\hline iRBC & Infected Red Blood Cell \\
\hline MRTC & Malaria Research and Training Center \\
\hline NETs & Neutrophil Extracellular Traps \\
\hline OR & Odds Ratio \\
\hline PA & Pyronaridine artesunate \\
\hline PCE & Parasite Clearance Estimator \\
\hline Plasmodium spp & Plasmodium all species \\
\hline PMN & Polymorphonuclear neutrophils \\
\hline $\mathrm{RBC}$ & Red blood cells \\
\hline ROS & Reactive Oxygen Species \\
\hline SSA & sub-Saharan Africa \\
\hline $\mathrm{TH}$ & T helper cell \\
\hline UNIKIN & University of Kinshasa \\
\hline USTTB & University of Sciences of Techniques and Technologies of Bamako \\
\hline$\mu \mathrm{L}$ & Microliter \\
\hline WANECAM & West African Network for Antimalarial Drugs \\
\hline WWARN & WorldWide Antimalarial Resistance Network \\
\hline
\end{tabular}

\section{Declarations}

\section{Ethics approval and consent to participate}

The West African Network for Antimalarial Drugs (WANECAM) protocol (IP_07_31060_002) was approved by local ethics committees for each site. All adults' patients provided signed informed consent before their participation in the study. For children, the consents were obtained from parents or legal guardians.

\section{Consent for publication}

Not applicable

\section{Availability of data and material}

The study is registered at the Pan African Clinical Trials Registry, number PACTR201105000286876. Registered 31 March 2011 
https://pactr.samrc.ac.za/Search.aspx

\section{Competing interests}

None of the authors declare a conflict of interests.

\section{Author's contributions}

Abdoulaye Djimde, Siriman Sodiomo, Jean Bosco Ouedraogo, Abdoul Habib Beavogui and Issaka Sagara were responsible for the conception and design of the clinical trial on which this study was based. Moussa Djimde, Kassoum Kayentao, Issaka Sagara, Alassane Dicko, Petra Mens, Henk Schallig and Abdoulaye Djimde conceived this analysis. Moussa Djimde did statistical analysis and wrote the first draft of the paper. All authors contributed to critical review and approved the final manuscript.

\section{Authors' information}

Moussa Djimde: mdjimde@icermali.org

Japhet Kabalu Tshiongo: kabalujaphet@gmail.com

Kassoum Kayentao: kayentao@icermali.org

Bakary Fofana: bfofana@icermali.org

Charles Arama: charama@icermali.org

Siriman Sodiomo: s.sirima@gras.bf

Jean Bosco Ouedraogo: jb_ouedraogo@hotmail.com

Abdoul Habib Beavogui: beavoguia_h@yahoo.fr

Issaka Sagara: isagara@icermali.org

Alassane Dicko: adicko@icermali.org

Petra Mens: p.f.mens@amsterdamumc.nl

Henk Schallig: h.d.schallig@amsterdamumc.nl

Abdoulaye Djimde: adjimde@icermali.org

\section{Acknowledgments}

We thank the fields, laboratories, data management and study admin staff at each of the seven trial sites in Burkina Faso, Guinea and Mali whose combined efforts made this work possible. We express our special thanks and gratitude to WANECAM for providing us the data that enabled this analysis.

\section{Funding}

The main clinical trial [Phase IIIb / IV, randomized, open, parallel to 3 arms and multicenter comparative clinical study comparing the efficacy and tolerance in repeated treatment of pyronaridine-artesunate and dihydroartemisinin-piperaquine with those of artesunate-amodiaquine and artemether-lumefantrine over a two-year period in children and adults with uncomplicated Plasmodium sp.] work was supported by the European and Developing Countries Clinical Trial Partnership (EDCTP), Medicines for Malaria Venture (MMV), the UK Medical Research Council, the Swedish International Development Cooperation Agency, German Ministry for Education and Research, University Claude Bernard (Lyon, France), University of Science, Techniques and Technologies of Bamako (Bamako, Mali), the Centre National de Recherche et de Formation sur le Paludisme (Burkina Faso), Institut de Recherche en Sciences de la Santé (Bobo-Dioulasso, Burkina Faso), and Centre National de Formation et de Recherche en Santé Rurale (Republic of Guinea).

This analysis was supported through the PYRAPREG project capacity building activities (PhD training). The PYRAPREG consortium is part of the EDCTP2 programme which is supported by the European Union under Horizon 2020.

\section{References}


1. Kolaczkowska E, Kubes P. Neutrophil recruitment and function in health and inflammation. Nat. Rev. Immunol. 2013.

2. Hsieh MM, Everhart JE, Byrd-Holt DD, Tisdale JF, Rodgers GP. Prevalence of neutropenia in the U.S. population: Age, sex, smoking status, and ethnic differences. Ann. Intern. Med. 2007.

3. Kondera-Anasz Z, Mielczarek-Palacz A, Switała J, Piasecka A. Metabolic activity of peripheral blood neutrophils in women with ovarian tumours. Ginekol. Pol. 2004.

4. Jenne CN, Wong CHY, Zemp FJ, McDonald B, Rahman MM, Forsyth PA, et al. Neutrophils recruited to sites of infection protect from virus challenge by releasing neutrophil extracellular traps. Cell Host Microbe. 2013.

5. Vazquez MI, Catalan-Dibene J, Zlotnik A. B cells responses and cytokine production are regulated by their immune microenvironment. Cytokine. 2015.

6. Kaiko GE, Horvat JC, Beagley KW, Hansbro PM. Immunological decision-making: How does the immune system decide to mount a helper Tcell response? Immunology. 2008.

7. Bajaj L, Hambidge S, Nyquist AC, Kerby G. Berman's Pediatric Decision Making. Berman's Pediatr. Decis. Mak. 2011.

8. Wood AJJ, Pizzo PA. Management of Fever in Patients with Cancer and Treatment-Induced Neutropenia. N. Engl. J. Med. 1993.

9. Donadieu J, Fenneteau O, Beaupain B, Mahlaoui N, Chantelot CB. Congenital neutropenia: Diagnosis, molecular bases and patient management. Orphanet J. Rare Dis. 2011.

10. Haddy TB, Rana SR, Castro O. Benign ethnic neutropenia: What is a normal absolute neutrophil count? J. Lab. Clin. Med. 1999.

11. Thobakgale CF, Ndung'U T. Neutrophil counts in persons of African origin. Curr. Opin. Hematol. 2014.

12. Miller LH, Mason SJ, Clyde DF, McGinniss MH. The resistance factor to Plasmodium vivax in blacks. The Duffy-blood-group genotype, FyFy. N. Engl. J. Med. 1976.

13. Yang CT, Cambier CJ, Davis JM, Hall CJ, Crosier PS, Ramakrishnan L. Neutrophils exert protection in the early tuberculous granuloma by oxidative killing of mycobacteria phagocytosed from infected macrophages. Cell Host Microbe. 2012.

14. Sun T, Chakrabarti C. Schizonts, merozoites, and phagocytosis in falciparum malaria. Ann. Clin. Lab. Sci. 1985.

15. Wickramasinghe SN, Phillips RE, Looareesuwan S, Warrell D a, Hughes M. The bone marrow in human cerebral malaria: parasite sequestration within sinusoids. Br. J. Haematol. 1987.

16. Kharazmi A, Jepsen S. Enhanced inhibition of in vitro multiplication of Plasmodium falciparum by stimulated human polymorphonuclear leucocytes. Clin. Exp. Immunol. 1984.

17. Brown J, Smalley ME. Inhibition of the in vitro growth of Plasmodium falciparum by human polymorphonuclear neutrophil leucocytes. Clin. Exp. Immunol. 1981.

18. Healer J, Graszynski A, Riley E. Phagocytosis does not play a major role in naturally acquired transmission-blocking immunity to Plasmodium falciparum malaria. Infect. Immun. 1999.

19. World Health Organisation(WHO). World Malaria Report 2012. http//www.who.int/malaria/publications/world_malaria_report_2012/en/; Consult. 17 March 2017. 2013.

20. Kokwaro G. Ongoing challenges in the management of malaria. Malar. J. [Internet]. 2009;8:S2. Available from: http://malariajournal.biomedcentral.com/articles/10.1186/1475-2875-8-S1-S2

21. Zwang J, Ndiaye JL, Djimdé A, Dorsey G, Mårtensson A, Karema C, et al. Comparing changes in haematologic parameters occurring in patients included in randomized controlled trials of artesunate-amodiaquine vs single and combination treatments of uncomplicated falciparum in sub-Saharan Africa. Malar. J. 2012.

22. Aitken EH, Alemu A, Rogerson SJ. Neutrophils and Malaria. Front. Immunol. 2018.

23. Sagara I, Beavogui AH, Zongo I, Soulama I, Borghini-Fuhrer I, Fofana B, et al. Pyronaridine-artesunate or dihydroartemisinin-piperaquine versus current first-line therapies for repeated treatment of uncomplicated malaria: a randomised, multicentre, open-label, longitudinal, controlled, phase 3b/4 trial. Lancet. 2018.

24. HORIBA. ABX Pentra 60. https//www.horiba.com/en_en/products/detail/action/show/Product/abx-pentra-60-584/; Accessed 23 Mai 2020.

25. Berliner N. Leukocytosis and Leukopenia. Goldman's Cecil Med. Twenty Fourth Ed. 2011.

26. World Health Organisation(WHO). Malaria Microscopy quality assurance manual.

https//apps.who.int/iris/bitstream/handle/10665/204266/9789241549394_eng.pdf;jsessionid=461F0A67D0C4CE83B811CE61858533BF? sequence=1; Accessed June 2020. 2016.

27. Olliaro P, Djimdé A, Dorsey G, Karema C, Mårtensson A, Ndiaye JL, et al. Hematologic parameters in pediatric uncomplicated Plasmodium falciparum malaria in sub-Saharan Africa. Am. J. Trop. Med. Hyg. 2011.

28. Berens-Riha N, Kroidl I, Schunk M, Alberer M, Beissner M, Pritsch M, et al. Evidence for significant influence of host immunity on changes in differential blood count during malaria. Malar. J. 2014. 
29. Ouologuem DT, Kone CO, Fofana B, Sidibe B, Togo AH, Dembele D, et al. Differential infectivity of gametocytes after artemisinin-based combination therapy of uncomplicated falciparum malaria. Afr. J. Lab. Med. 2018.

30. Andersohn F, Konzen C, Garbe E. Systematic review: Agranulocytosis induced by nonchemotherapy drugs. Ann. Intern. Med. 2007.

31. Kaufman DW, Kelly JP, Issaragrisil S, Laporte JR, Anderson T, Levy M, et al. Relative incidence of agranulocytosis and aplastic anemia. Am. J. Hematol. 2006.

32. Adjei GO, Kurtzhals JAL, Rodrigues OP, Alifrangis M, Hoegberg LCG, Kitcher ED, et al. Amodiaquine-artesunate vs artemether-lumefantrine for uncomplicated malaria in Ghanaian children: A randomized efficacy and safety trial with one year follow-up. Malar. J. 2008.

33. Novartis. Drug Regulatory Affairs Coartem ${ }^{\circledR} /$ Riamet ${ }^{\circledR}$ Basic Prescribing Information. 2007.

34. D'Alessandro U. DIHYDROARTEMISININ-PIPERAQUINE: AN UPDATE. Parasitol. Inst. Trop. Med. Antwerp, Flanders [Internet]. Belgium; 2010. Available from: https://www.dndi.org/wp-content/uploads/2010/08/dalessandro.pdf, Accessed 29 April 2020

35. Winstanley P, Edwards G, Orme M, Breckenridge A. The disposition of amodiaquine in man after oral administration. Br. J. Clin. Pharmacol. 1987.

36. Krishna S, White NJ. Pharmacokinetics of Quinine, Chloroquine and Amodiaquine: Clinical Implications. Clin. Pharmacokinet. 1996.

37. Ramanathan S, Karupiah S, Nair NK, Olliaro PL, Navaratnam V, Wernsdorfer WH, et al. A new and simple solid-phase extraction method for LC determination of pyronaridine in human plasma. J. Chromatogr. B Anal. Technol. Biomed. Life Sci. 2005.

38. WWARN Gametocyte Study Group. Gametocyte carriage in uncomplicated Plasmodium falciparum malaria following treatment with artemisinin combination therapy: a systematic review and meta-analysis of individual patient data. BMC Med. [Internet]. 2016;14:79. Available from: http://bmcmedicine.biomedcentral.com/articles/10.1186/s12916-016-0621-

7\%5Cnhttp://www.ncbi.nlm.nih.gov/pubmed/27221542\%5Cnhttp://www.pubmedcentral.nih.gov/articlerender.fcgi?artid=PMC4879753

39. Flegg JA, Guérin PJ, Nosten F, Ashley EA, Phyo AP, Dondorp AM, et al. Optimal sampling designs for estimation of Plasmodium falciparum clearance rates in patients treated with artemisinin derivatives. Malar. J. 2013.

\section{Tables}

Table 1: Baseline characteristics according to neutrophils status 


\begin{tabular}{|c|c|c|c|c|c|}
\hline \multicolumn{2}{|c|}{ Baseline characteristics } & \multirow{2}{*}{$\begin{array}{l}\text { Neutropenia } \\
\mathrm{N}=1226 \\
\mathrm{n}(\%)\end{array}$} & \multirow{2}{*}{$\begin{array}{l}\text { Normal count } \\
\mathrm{N}=1792 \\
\mathrm{n}(\%)\end{array}$} & \multirow{2}{*}{$\begin{array}{l}\text { Neutrophilia } \\
N=1827 \\
n(\%)\end{array}$} & \multirow[t]{2}{*}{$\mathrm{p}$-value } \\
\hline & & & & & \\
\hline \multirow[t]{4}{*}{ Treatment arms } & $\mathrm{AL}(\mathrm{n}=1567)$ & $321(20.48)$ & $602(38.42)$ & $644(41.10)$ & \multirow[t]{4}{*}{$<0.0001$} \\
\hline & AS-AQ $(n=961)$ & $264(27.47)$ & $340(35.38)$ & $357(37.15)$ & \\
\hline & DHA-PQ $(n=1154)$ & $316(27.38)$ & $401(34.75)$ & $437(37.87)$ & \\
\hline & $\mathrm{PA}(\mathrm{n}=1163)$ & 325 (27.94) & $449(38.61)$ & $389(33.45)$ & \\
\hline \multirow[t]{3}{*}{ Age categories } & $<5$ years $(n=1165)$ & $409(35.11)$ & $460(39.48)$ & $296(25.41)$ & \multirow[t]{3}{*}{$<0.0001$} \\
\hline & 5 to 14 y $(n=2981)$ & $704(23.62)$ & $1105(37.07)$ & $1172(39.31)$ & \\
\hline & $>14$ y $(n=699)$ & $113(16.17)$ & $227(32.47)$ & 359 (51.36) & \\
\hline \multirow[t]{2}{*}{ Patient gender } & Male $(n=2489)$ & $633(25.43)$ & $908(36.48)$ & $948(38.09)$ & \multirow[t]{2}{*}{0.7476} \\
\hline & Female $(n=2356)$ & $593(25.17)$ & $884(37.52)$ & $879(37.31)$ & \\
\hline \multirow[t]{3}{*}{ Countries } & Burkina Faso $(n=1739)$ & $330(18.98)$ & $674(38.76)$ & 735 (42.26) & \multirow[t]{3}{*}{$<0.0001$} \\
\hline & Guinea $(n=770)$ & $340(44.04)$ & $245(31.74)$ & $185(24.22)$ & \\
\hline & Mali $(n=2334)$ & $556(23.82)$ & $873(37.40)$ & 905 (38.78) & \\
\hline \multicolumn{2}{|l|}{ Gametocytemia } & $117(35.67)$ & $133(40.55)$ & 78 (23.78) & $<0.0001$ \\
\hline \multicolumn{2}{|c|}{ Patients weight $(\mathrm{Kg})$ : median (interquartile range) } & $19.55(14.0,28.8)$ & $21.80(15.8,32.7)$ & $24.50(17.6,37.8)$ & $<0.0001$ \\
\hline \multicolumn{2}{|c|}{ Temperature $\left({ }^{\circ} \mathrm{C}\right)$ : median (interquartile range) } & $36.9(36.5,37.5)$ & $37.5(36.8,38.3)$ & $38.2(37.4,38.9)$ & $<0.0001$ \\
\hline \multicolumn{2}{|c|}{ P. falciparum $(/ \mu \mathrm{l})$ : median (interquartile range) } & $2590(520,19320)$ & $15200(1060,46475)$ & $29760(10310,60320)$ & $<0.0001$ \\
\hline \multicolumn{2}{|c|}{ Haemoglobin $(\mathrm{g} / \mathrm{dl})$ : median (interquartile range) } & $10.1(9.0,11.1)$ & $10.6(9.6,11.6)$ & $11.0(10.1,11.9)$ & $<0.0001$ \\
\hline \multicolumn{2}{|c|}{ White Blood Cell: median (interquartile range) } & $6.5(5.0,8.6)$ & $6.9(5.2,8.7)$ & $8.0(6.3,10.6)$ & $<0.0001$ \\
\hline \multicolumn{2}{|c|}{ Lymphocytes: median (interquartile range) } & $48.60(42.92,53.7)$ & $32.55(27.00,38.2)$ & $15.50(8.10,20.3)$ & $<0.0001$ \\
\hline
\end{tabular}

$\mathrm{N}$ : frequency; PA: Pyronaridine artesunate; DHA-PQ: Dihydroartemisinin piperaquine;

AL: Artemether lumefantrine; AS-AQ: Artesunate amodiaquine; Q1: $25^{\text {th }}$ percentile; Q3: $75^{\text {th }}$ percentile

Table 2: Proportion of patients with parasitaemia at days 3,7 and 28 at the first episode 


\begin{tabular}{|c|c|c|c|c|}
\hline Patients with parasitaemia & $\begin{array}{l}\text { Neutropenia } \\
\mathrm{n}(\%)\end{array}$ & $\begin{array}{l}\text { Normal count } \\
\mathrm{n}(\%)\end{array}$ & $\begin{array}{l}\text { Neutrophilia } \\
\mathrm{n}(\%)\end{array}$ & $\mathrm{p}$-value \\
\hline \multicolumn{5}{|l|}{ Artemether Lumefantrine } \\
\hline Day 3 & $2(100.00)$ & $0(0.00)$ & $0(0.00)$ & - \\
\hline Day 7 & $4(100.00)$ & $0(0.00)$ & $0(0.00)$ & - \\
\hline Day 28 & $85(50.90)$ & $72(43.11)$ & $10(5.99)$ & $<0.0001$ \\
\hline \multicolumn{5}{|l|}{ Artesunate Amodiaquine } \\
\hline Day 3 & $1(100.00)$ & $0(0.00)$ & $0(0.00)$ & - \\
\hline Day 7 & $0(0.00)$ & $0(0.00)$ & $0(0.00)$ & - \\
\hline Day 28 & $20(58.82)$ & $10(29.41)$ & $4(11.77)$ & $<0.0001$ \\
\hline \multicolumn{5}{|c|}{ Dihydroartemisinin Piperaquine } \\
\hline Day 3 & $2(100.00)$ & $0(0.00)$ & $0(0.00)$ & - \\
\hline Day 7 & $0(0.00)$ & $0(0.00)$ & $0(0.00)$ & - \\
\hline Day 28 & $5(100.00)$ & $0(0.00)$ & $0(0.00)$ & - \\
\hline \multicolumn{5}{|l|}{ Pyronaridine Artesunate } \\
\hline Day 3 & $1(100.00)$ & $0(0.00)$ & $0(0.00)$ & - \\
\hline Day 7 & $0(0.00)$ & $0(0.00)$ & $0(0.00)$ & - \\
\hline Day 28 & $11(73.33)$ & $4(26.67)$ & $0(0.00)$ & $<0.0001$ \\
\hline
\end{tabular}

Table 3: Logistic regression model showing the effect of socio-demographic, biological parameters and treatment used on malaria parasite reappearance at Day 28

\begin{tabular}{|lllll|}
\hline & OR & \multicolumn{2}{l}{$95 \% \mathrm{Cl}$} & \multirow{2}{*}{ p-value } \\
\cline { 3 - 5 } & & Lower & Upper & \\
\cline { 1 - 5 } Neutrophils normal & 0.97 & 0.37 & 2.53 & 0.9471 \\
\hline Neutrophilia & 1.57 & 0.17 & 13.09 & 0.6771 \\
\hline AS-AQ & 0.27 & 0.11 & 0.62 & 0.0024 \\
\hline DHA-PQ & 0.07 & 0.02 & 0.62 & $<0.0001$ \\
\hline PA & 0.13 & 0.05 & 0.33 & $<0.0001$ \\
\hline Lymphocytes & 0.93 & 0.89 & 0.98 & 0.0063 \\
\hline Haemoglobin & 1.04 & 0.78 & 1.39 & 0.8059 \\
\hline Sex Male & 0.57 & 0.29 & 1.11 & 0.1036 \\
\hline Under 5 & 5.64 & 1.33 & 40.94 & 0.0393 \\
\hline Age: 5 to 14 years & 3.38 & 0.85 & 23.72 & 0.1357 \\
\hline
\end{tabular}

AS-AQ: Artesunate-amodiaquine; DHA-PQ: Dihydroartemisinin piperaquine; PA: Pyronaridine-artesunate

Figures 
Artemether-lumefantrine 0.80 Artesunate-amodiaquine 1.73 DHA-piperaquine 0.99

Pyronaridine-artesunate 0.97 Burkina

Guinea

Mali

Male

Female

Under5

Teenager

Anemia

Fixed effect model

0.88

0.29

1.51

0.56

1.66

0.48

0.56

1.12

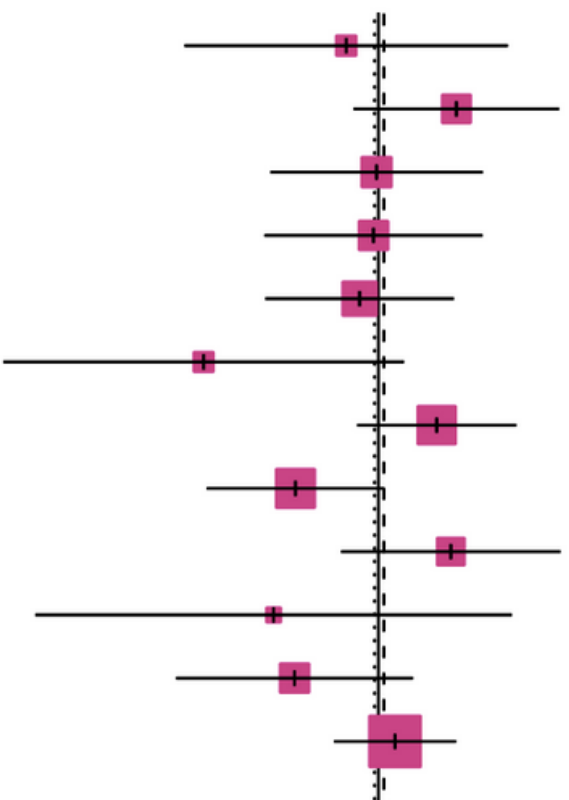

Random effects model
1.04 0.97

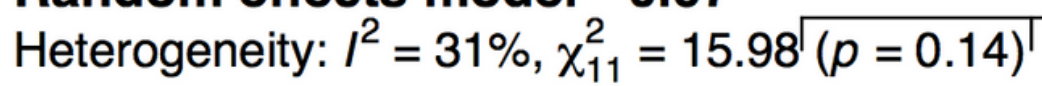

0.1

$\begin{array}{lll}0.5 & 12\end{array}$

$[0.26 ; 2.46]$

[0.85; 3.52] $[0.47 ; 2.06]$ $[0.45 ; 2.06]$ $[0.46 ; 1.68]$ $[0.07 ; 1.19]$ $[0.87 ; 2.61]$ $[0.30 ; 1.03]$ $[0.78 ; 3.55]$ $[0.09 ; 2.53]$ $[0.24 ; 1.26]$ $[0.74 ; 1.71]$

$[0.85 ; 1.27]$ [0.75; 1.26]

Figure 1

Prevalence of gametocytemia before treatment and on post treatment days 3,7 and 28 at the first episode 


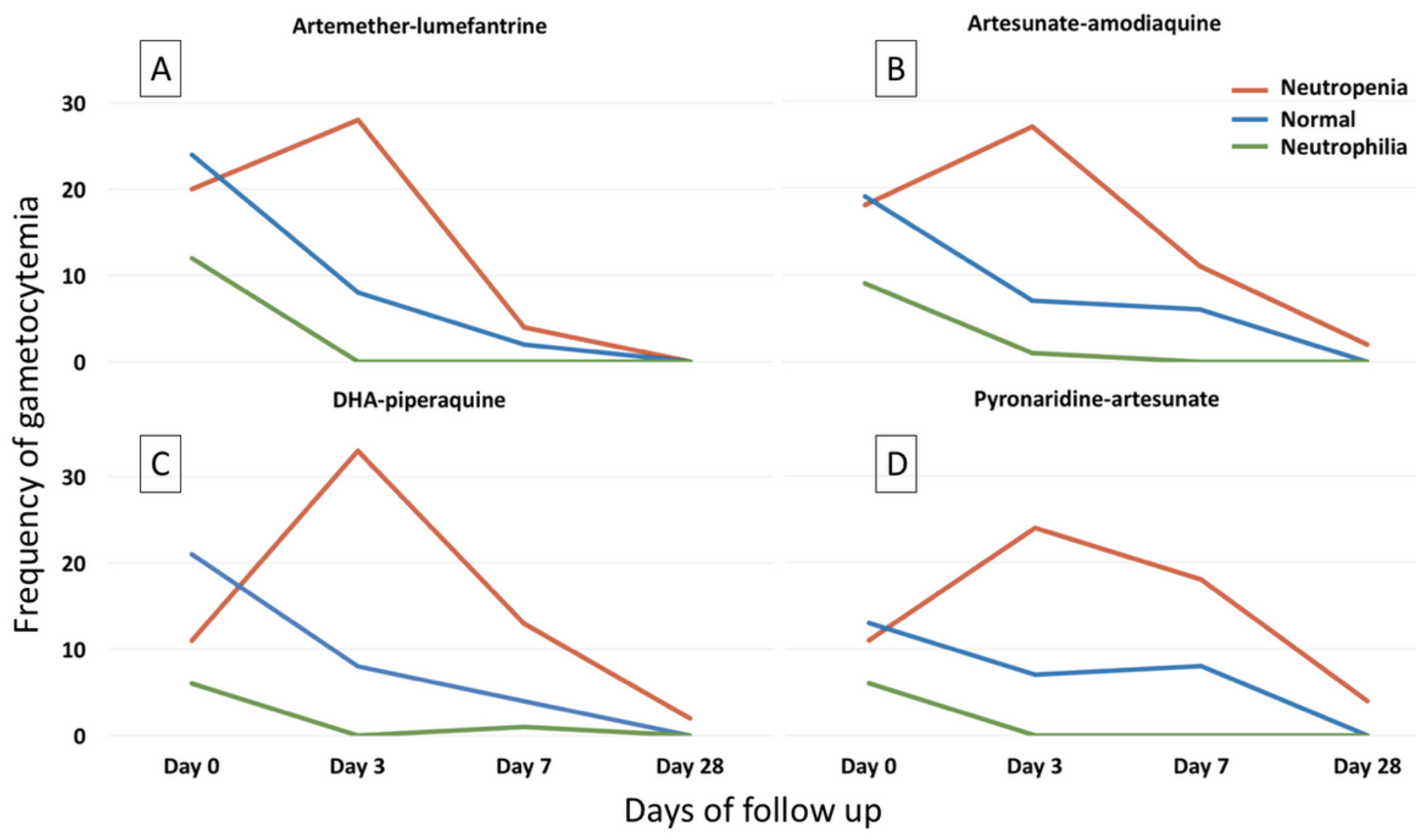

Figure 2

A forest plot investigating the association among socio-demographic parameters and treatment used on gametocytes reappearance at Day 3 according to neutrophils variation

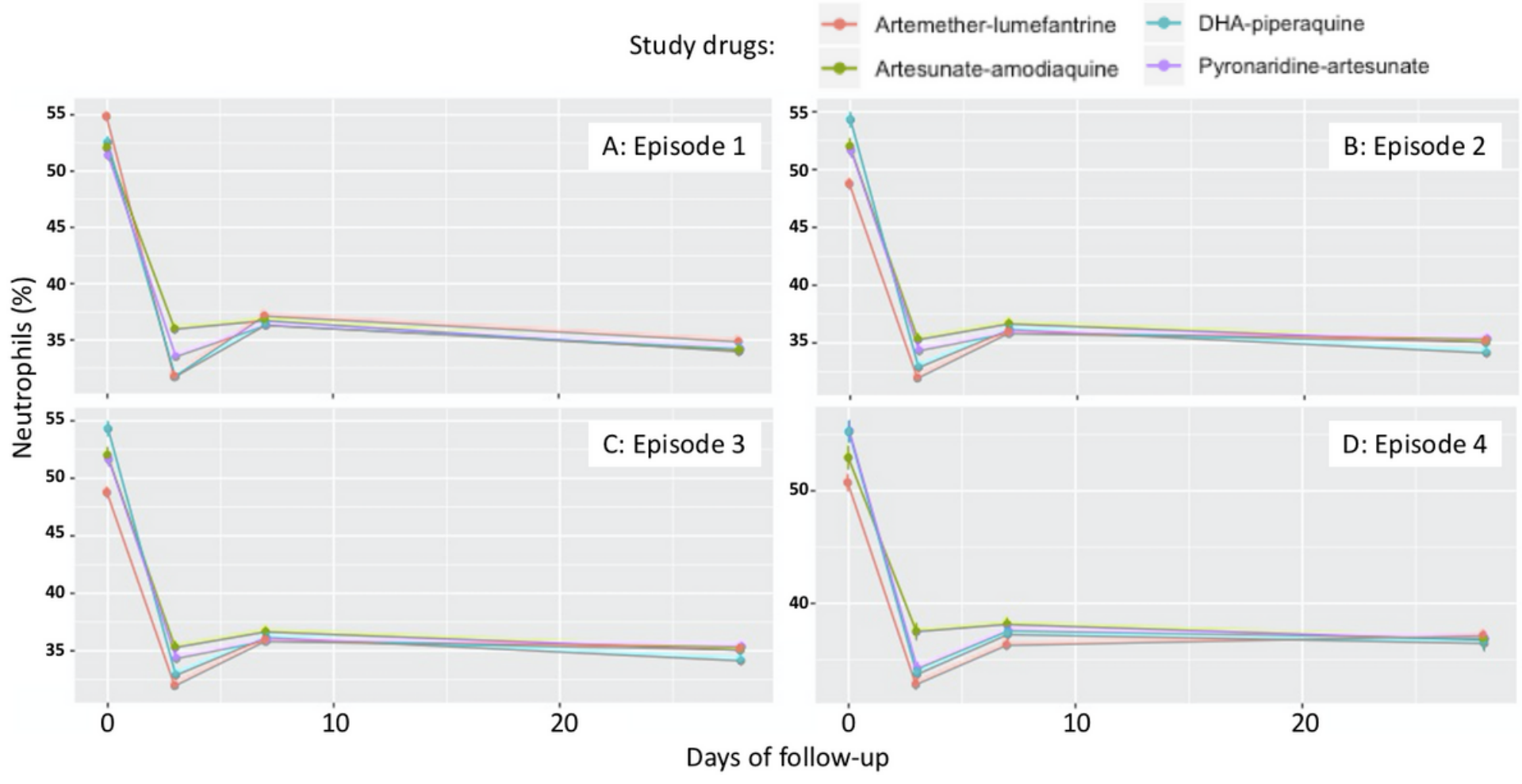

Figure 3

Comparison of post treatment neutrophils levels means between treatment arms 